Fig. 1.-Antero-posterior chest radiograph of $\mathbf{K}$ Case 1.

\title{
TOTAL ANOMALOUS PULMONARY VENOUS DRAINAGE WITH UNUSUAL FEATURES
}

\author{
BY \\ A. W. VENABLES, P. E. CAMPBELL, AND G. W. WESTLAKE \\ From the Cardiac Department, Royal Children's Hospital, Melbourne, Victoria, Australia \\ Received April 29, 1963
}

The occurrence of relatively soft continuous murmurs of the venous hum type in supradiaphragmatic forms of total anomalous pulmonary venous drainage is well recognized (Keith et al., 1954; Keith, Rowe, and Vlad, 1958). Loud continuous murmurs occur at times but are considerabiy less common (McKusick, 1958). This paper describes two cases of total anomalous pulmonary venous drainage in which loud continuous murmurs simulating those of patent ductus arteriosus were heard in the left infraclavicular region. These murmurs appear to have been caused by unusual anatomical variations in the anomalous vertical venous channels by which pulmonary venous blood drained to the superior vena cava.

\section{CAse Reports}

Case 1. A baby girl (birth weight was $3 \cdot 1 \mathrm{~kg}$.) aged 3 days was admitted in December 1962 with cyanosis and umbilical cord hæmorrhage. There was no relevant antenatal or natal history. She was initially only mildly cyanosed but deteriorated progressively with increasing cyanosis and dyspnoea, and finally restlessness. Death occurred on the twentieth day of life.

At 3 days there was a continuous murmur at the pulmonary area, which was relatively soft but later became quite loud and of a quality simulating that of a patent ductus. Radiographs showed a fairly normal cardiac contour with pulmonary congestion but no clear evidence of pulmonary œdema (Fig. 1). The electrocardiogram showed upright $T$ waves in the right chest leads, but was otherwise normal for the baby's age. Cardiac catheterization at the age of 9 days demonstrated total anomalous pulmonary venous drainage to the superior vena cava. The catheter entered the anomalous pulmonary venous channel at the left of the mediastinum, but did not pass beyond the left main bronchus (Fig. 2). The right ventricular systolic pressure was $70 \mathrm{~mm}$. Hg. Oxygen saturations suggested that pulmonary flow was reduced (Table I). Selective angiogtaphy was confined to injection into the left atrium via the atrial septal communication.

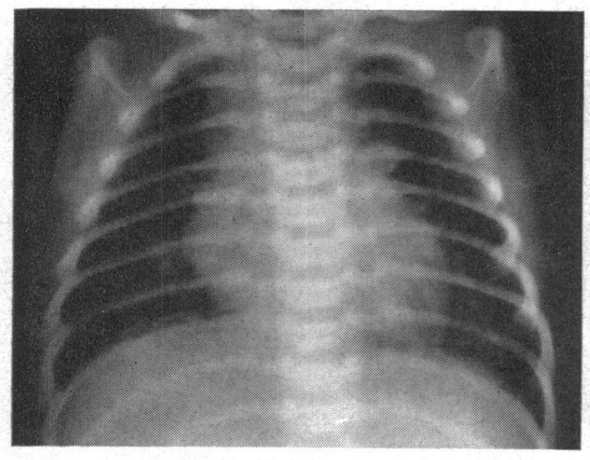

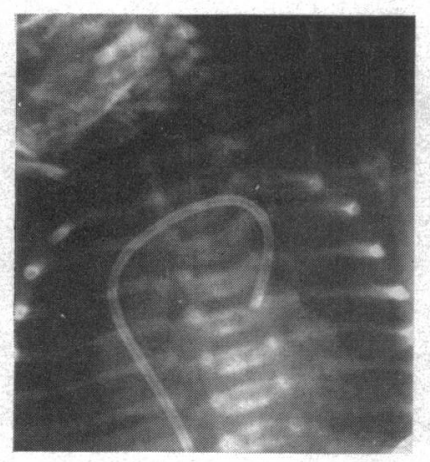

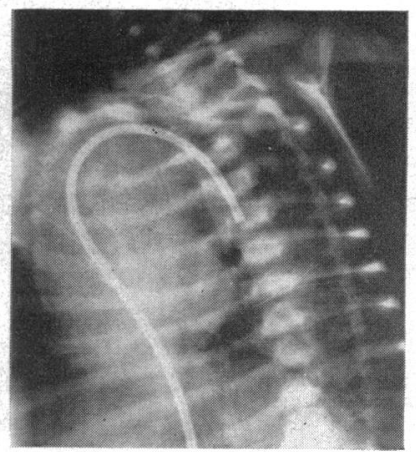

FIG. 2.-Antero-posterior and left anterior oblique views of 129 catheter in vertical vein, in Case 1. 
TABLE I

Cardiac Catheterization Data

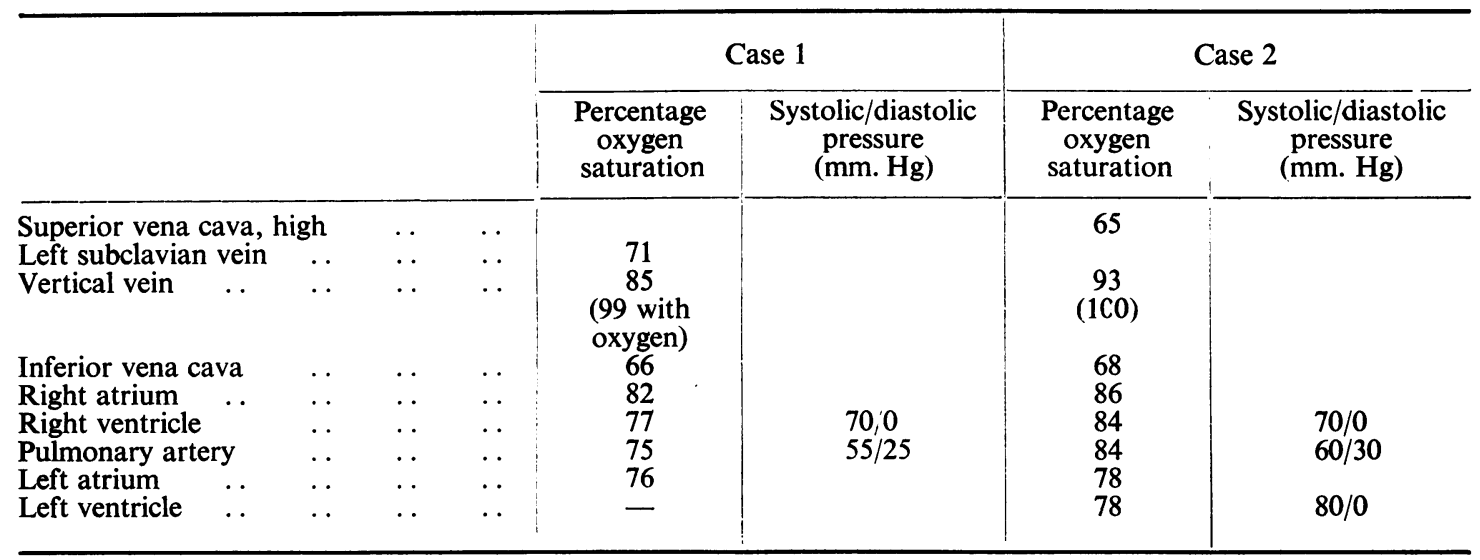

Necropsy. The significant findings were confined to the respiratory and cardiovascular systems. The lungs showed much congestion and patchy bronchopneumonic consolidation, and under the microscope many alveoli were seen to be stuffed with siderophages. No significant changes were present in the pulmonary arterioles.

The heart was of normal size and the cardiothoracic ratio (maximum transverse diameters) was 5:11. The heart was somewhat cone-shaped and lay transversely in the mediastinum.

The pulmonary venous return was abnormal. The striking feature on opening the thorax was the presence of a large venous trunk that appeared between the left lung and left pulmonary artery and curved upwards and medially in front of the aorta, describing an arc as it crossed the midline to enter the right superior vena cava (Fig. 3). It was joined in its course by the left innominate vein.. The heart, lungs, and great vessels were then removed and perfused with formalin from a bottle suspended $15 \mathrm{~cm}$. above the bench. The subsequent description refers to the fixed, perfused specimen.

Further dissection showed that the trunk arose behind the heart from the union of the upper, middle, and lower pulmonary veins of the right lung. Shortly after its origin, it gave off a small branch which passed through the diaphragm to join the portal vein in the porta hepatis (Fig. 4).

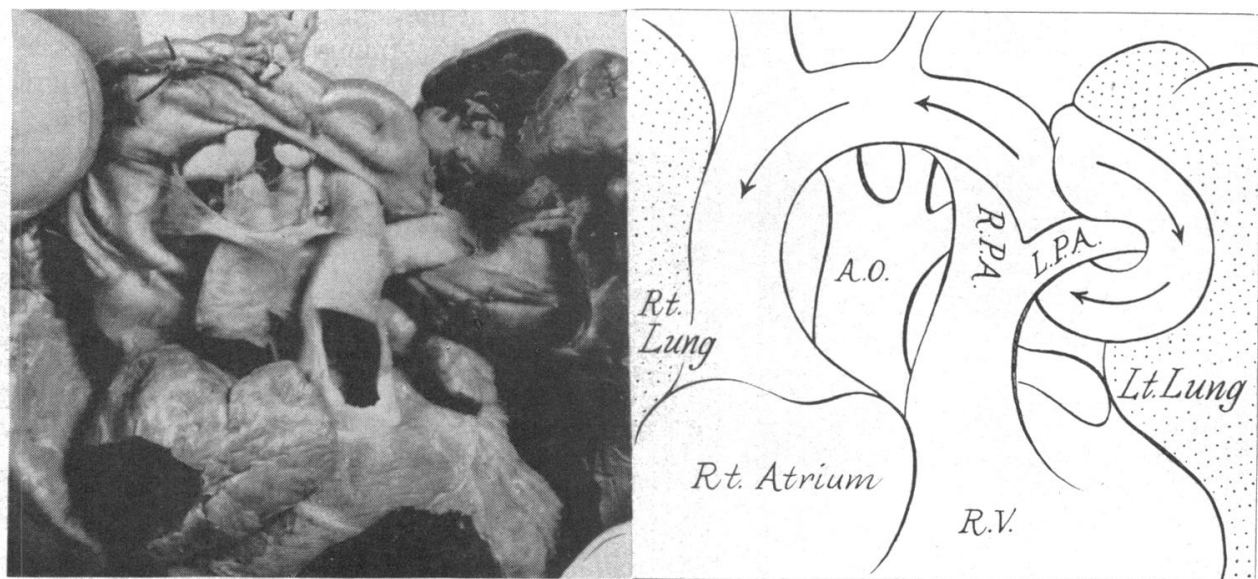

FIG. 3.-Photograph and schematic diagram of the anterior view of the heart and great vessels showing the anomalous venous trunk entering the right atrium. The vessels have been stretched to the right to display the course of the anomalous trunk (Case 1). 
The common trunk then passed transversely behind the heart without communicating with the left atrium and became partly buried in the substance of the left lung. The trunk then passed upwards almost completely buried in the lung substance receiving the left pulmonary veins in its course (Fig. 5). It then curved forward over the top of the left pulmonary artery and could be seen emerging anteriorly from the lung substance in front of the pulmonary artery and bronchus (Fig. 3). The wall here was thin, and the lumen dilated. The trunk then dived between left pulmonary artery and left main bronchus, where it was moderately constricted, coming up on the posterior aspect of the left pulmonary artery which was thus completely encircled. From this point it passed upwards, lateral to the ductus and anterior to the aorta, to enter the superior vena cava. Just before the insertion of the left innominate vein, the vessel narrowed from $1 \mathrm{~cm}$. to $0.4 \mathrm{~cm}$. diameter.

The foramen ovale and ductus arteriosus were patent. The tricuspid and pulmonary valves were anatomically normal, as were the mitral and aortic valves, although the latter were small (internal diameters 0.9 and $0.7 \mathrm{~cm}$. respectively). The right ventricle was hypertrophied (wall thickness $0.5 \mathrm{~cm}$.); the left ventricle was small but otherwise normal (wall thickness $0.6 \mathrm{~cm}$.).

Case 2. A boy aged 20 months was admitted in November 1962 after referral from the Brisbane Children's Hospital. He was stated to have been somewhat blue from birth, with recurrent severe respiratory infections and episodes of cardiac failure. Weight gains had been poor, and his weight on admission was $10 \cdot 2 \mathrm{~kg}$.

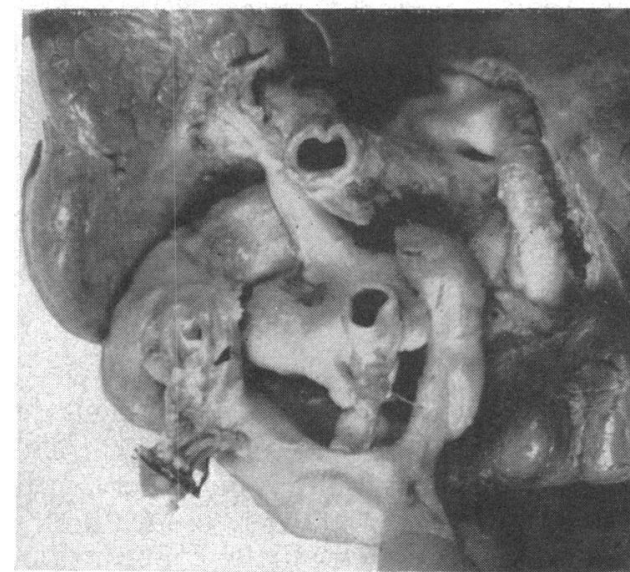

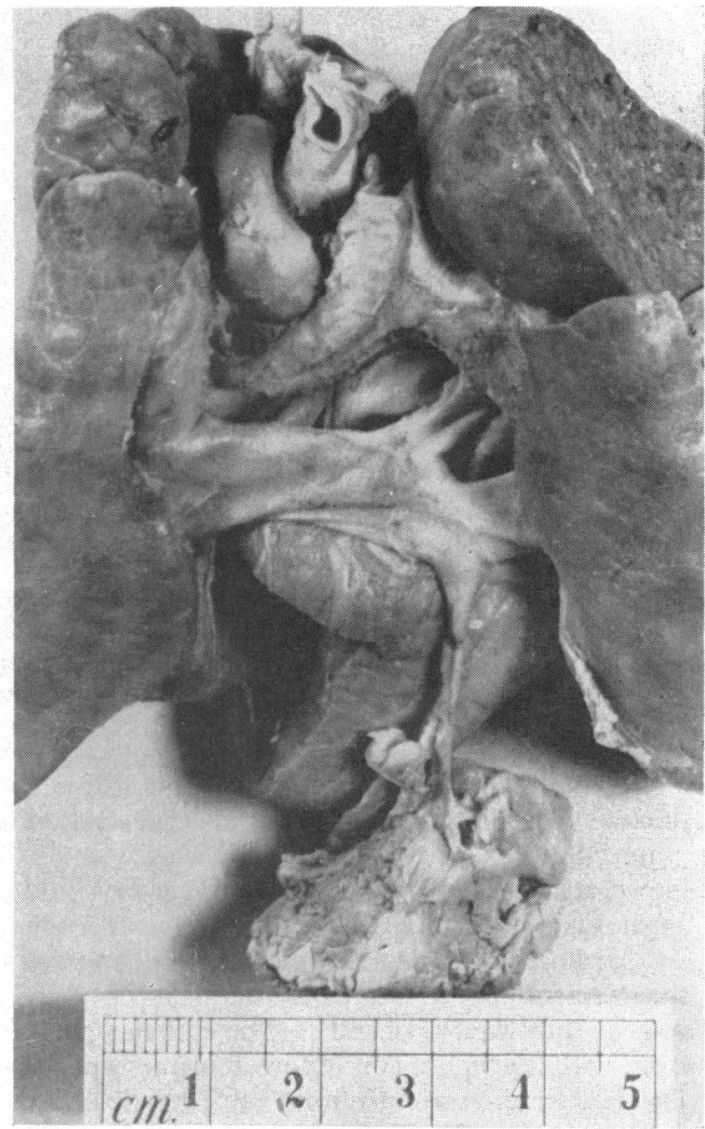

FIG. 4.-View of the posterior surface of the heart, showing the origin of the venous confluence and the small branch passing to the portal vein (Case 1).

FIG. 5.-Photograph and schematic diagram of the superior aspect of the great vessels, showing the helical course of the vertical vein. The first constriction where the vessel passes between pulmonary artery and bronchus is not easily seen due to stretching of the structures during dissection, but the second constriction, just before the entry of the left innominate vein, is clearly seen (upper part of illustration is posterior). Case 1 . 


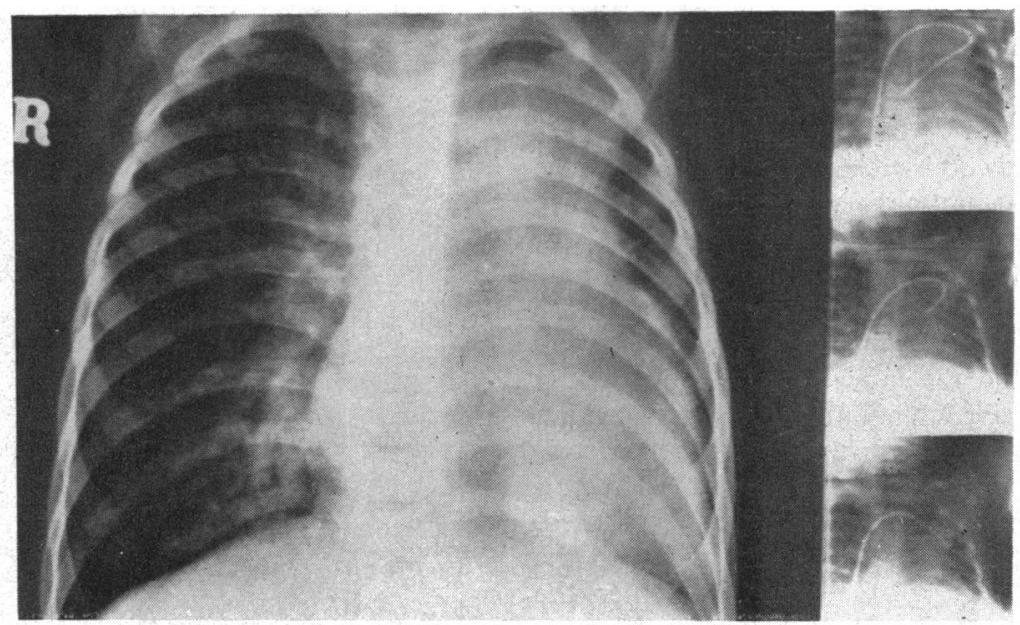

FIG. 6.-(A) Antero-posterior chest radiograph (Case 2). (B) Inserts show the catheter in three positions in anomalous pulmonary venous channel.

Examination revealed a dusky child with a loud continuous murmur of similar type to that of patent ductus in the left infraclavicular area, associated with obvious accentuation of pulmonary closure. Radiograph showed a large heart, with a substantial opacity at the left upper mediastinal border, previously interpreted as the main pulmonary artery, and with considerable pulmonary plethora (Fig. 6A). The cardiogram showed right ventricular hypertrophy.

Cardiac catheterization revealed total anomalous pulmonary venous drainage to the superior vena cava, the opacity at the left upper border of the mediastinum being due to aneurysmal dilatation of the left vertical vein at that level (Fig. 6B). The catheter passed only to the mid-line in the anomalous venous channel, from within which a continuous murmur was recorded. There was evidence of interatrial communication. Detailed findings are given in Table I. Selective angiography performed successively from left atrium and from right ventricle delineated the left heart but did not demonstrate the pulmonary venous system satisfactorily.

Repair of the lesion was performed successfully in December 1962 using cardiopulmonary bypass with moderate hypothermia (G.W.W.). The origin of the left vertical vein from the transverse common pulmonary vein was stenotic, appearing to be only about $4 \mathrm{~mm}$. in external diameter. There was considerable post-stenotic dilatation of the vertical vein above this point, this vessel measuring $3 \mathrm{~cm}$. in diameter. A continuous thrill was felt over the dilated segment. The transverse pulmonary vein was anastomosed to the posterior wall of the left atrium from outside the heart, an atrial septal defect approximately $2 \mathrm{~cm}$. in diameter having first been closed through the incision made in the left atrial wall for the anastomosis. The vertical vein was then ligated. Total duration of bypass was $\mathbf{5 0}$ minutes. The procedure was well tolerated: the immediate post-operative period was covered by assisted positive pressure respiration through a tracheostomy. Convalescence was uneventful, and the child was well when discharged home from hospital on the twelfth day after operation.

\section{Discussion}

Although the continuous murmurs present in these two patients simulated patent ductus with left-to-right shunt, other features, in particular cyanosis and evidence of right ventricular hypertrophy, excluded this diagnosis. In both cases the investigatory findings indicated that the clinical evidence of bidirectional shunting was due to total anomalous pulmonary venous drainage to the superior vena cava via a left vertical vein. These findings were essentially confirmed at necropsy in Case 1 and at operation in Case 2. Case 1 was demonstrated to have a small accessory channel from the common pulmonary vein to the portal vein in addition to the major drainage by the vertical vein. 
Intravascular phonocardiography demonstrated the presence of a continuous murmur in the vertical vein in Case 2 , but the abnormal anatomy underlying the loud continuous murmurs was not appreciated at the time of investigation in either of them. In one, no attempt was made to define the anatomy of the pulmonary venous drainage by forward angiography from the pulmonary artery or right ventricle, while in the other this procedure was unsatisfactory. In neither case did the catheter pass through the transverse common pulmonary vein to the right pulmonary veins as it frequently does once the vertical vein is entered. In retrospect this can be explained by the constrictions subsequently identified, but the presence of pulmonary venous obstruction was neither suspected nor identified at that time, wedged pulmonary artery pressures being unobtainable.

The continuous murmurs appear to have been related to the constrictions present in the ascending vertical pulmonary venous trunks. In Case 1 there were two constrictions of this channel along its most unexpected course, the first constriction being at the site of compression of the vessel between left pulmonary artery and left main bronchus, and the second being in the vessel wall more proximally. In Case 2 , the vertical channel was narrowed at its origin from the common pulmonary vein. Both cases exhibited post-stenotic dilatation of the vertical channel. A thrill was palpable over this dilatation at thoracotomy in Case 2.

The murmurs ordinarily heard in the unobstructed variety of total anomalous pulmonary venous drainage to the superior vena cava are soft venous hums (Keith et al., 1954; 1958). The increased intensity of the murmurs in the two cases reported appears to be due to the presence of the constrictions described, since the location of the murmurs was in keeping with this. The case described by McKusick (1958) exhibited a duct-like murmur to the right of the upper sternum: there was no evidence of stenosis, and the intensity. of the murmur was implied to be due to unusual turbulence in the very dilated superior vena cava.

Elliott and Edwards (1962) classified total anomalous pulmonary venous drainage to the superior vena cava into two types. In the first, the vertical vein passes anterior to the left pulmonary artery, while in the second the vertical vein ascends between the left pulmonary artery and the left main bronchus at which site it is subject to local constriction by the extrinsic pressure of these structures. In the two infants with this abnormality described by Kauffman, Ores, and Andersen (1962), there was gross narrowing of the vertical vein at this point with severe pulmonary venous obstruction and consequent pulmonary œdema. There was considerable local dilatation beyond the point of narrowing. Continuous murmurs were not present. Localized anatomical narrowing of the vertical trunk unrelated to such a course has been described by Hastreiter et al. (1962).

In Case 1 of the present report, the vertical vein followed an unusual course quite unlike any previously reported. From the venous confluence, the channel ascended first in the substance of the left lung behind the left main bronchus. It then encircled the pulmonary artery, finally passing upwards between pulmonary artery and bronchus from below in the manner of the course described by Elliott and Edwards (1962). Local narrowing was present at that level as in the previous cases, but additional local anatomical narrowing was present proximal to this. Retrospective assessment suggests the presence of considerable pulmonary venous obstruction.

Case 2 of this report is anatomically similar to Case 4 of Hastreiter et al. (1962). Their patient exhibited " rather prominent" narrowing of the vertical vein at its origin with aneurysmal dilatation of the vein itself, and is described as having a soft continuous murmur at the upper left sternal edge: death occurred at the age of 14 days from the characteristic syndrome of pulmonary venous obstruction. This contrasts with the present patient who presented in the usual manner of unobstructed supradiaphragmatic total anomalous pulmonary venous drainage with failure to thrive, recurrent respiratory infections, and heart failure without pulmonary œedema, indicating that the local obstruction was much less significant. A larger pulmonary venous return may explain the greater intensity of the murmur.

The cases reported here show that the presence of a loud continuous murmur at the left sternal edge in association with total anomalous pulmonary venous drainage to the superior vena cava 
should suggest unusual anatomy of the vertical venous channel with local narrowing of that structure of some type, even in the absence of overt evidence of pulmonary venous obstruction. Failure to pass a catheter into and through the transverse common pulmonary vein may be significant. Demonstration of the detailed anatomy of the pulmonary venous drainage in such cases should be possible by appropriate angiographic techniques.

Case 2 confirms the applicability of bypass operations to this condition.

\section{SUMMARY}

Two unusual cases of total anomalous pulmonary venous drainage exhibited loud continuous murmurs at the upper left sternal edge. These were apparently related to local constrictions in the course of the anomalous vertical channel that carried pulmonary venous blood to the superior vena cava via the innominate vein. Necropsy performed in one neonatal death demonstrated that the constrictions were associated with a previously undescribed bizarre course of the vertical vein. The findings were made in the second case during successful corrective surgery.

Case 2 was referred by Dr. E. G. Galea, Honorary Cardiologist to the Brisbane Children's Hospital: Dr. H. G. Hiller performed the cardiac catheterization in this case.

\section{REFERENCES}

Elliott, L. P., and Edwards, J. E. (1962). The problem of pulmonary venous obstruction in total anomalous pulmonary venous connection to the left innominate vein. (Editorial). Circulation, 25, 913.

Hastreiter, A. R., Paul, M. H., Molthan, M. E., and Miller, R. A. (1962). Total anomalous pulmonary venous connection with severe pulmonary venous obstruction. Circulation, 25, 916.

Kauffman, S. L., Ores, C. N., and Andersen, D. H. (1962). Two cases of total anomalous pulmonary venous return of the supracardiac type with stenosis simulating infradiaphragmatic drainage. Circulation, 25, 376.

Keith, J. D., Rowe, R. D., and Vlad, P. (1958). Heart Disease in Infancy and Childhood. Macmillan, New York. $-, \frac{16}{16}, 23$, and O'Hanley, J. H. (1954). Complete anomalous pulmonary venous drainage. Amer. J. Med.,

McKusick, V. (1958). Cardiovascular Sound in Health and Disease. Williams and Wilkins, Baltimore. 